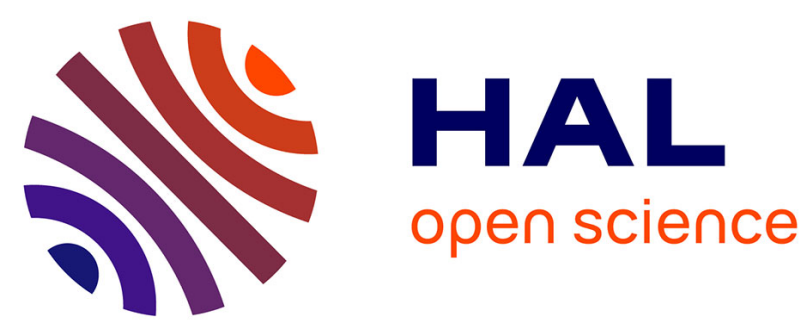

\title{
Three-dimensional reconstruction of a random fibrous medium: Geometry, transport, and sound absorbing properties
}

Hoang Tuan Luu, Camille Perrot, Vincent Monchiet, Raymond Panneton

\section{- To cite this version:}

Hoang Tuan Luu, Camille Perrot, Vincent Monchiet, Raymond Panneton. Three-dimensional reconstruction of a random fibrous medium: Geometry, transport, and sound absorbing properties. Journal of the Acoustical Society of America, 2017, 141 (6), pp.4768 - 4780. 10.1121/1.4989373 . hal-01580012

\section{HAL Id: hal-01580012 \\ https://hal.science/hal-01580012}

Submitted on 1 Sep 2017

HAL is a multi-disciplinary open access archive for the deposit and dissemination of scientific research documents, whether they are published or not. The documents may come from teaching and research institutions in France or abroad, or from public or private research centers.
L'archive ouverte pluridisciplinaire HAL, est destinée au dépôt et à la diffusion de documents scientifiques de niveau recherche, publiés ou non, émanant des établissements d'enseignement et de recherche français ou étrangers, des laboratoires publics ou privés. 


\title{
Three-dimensional reconstruction of a random fibrous medium: Geometry, transport, and sound absorbing properties
}

\author{
Hoang Tuan Luu ${ }^{\text {a) }}$ \\ Groupe d'Acoustique de l'Université de Sherbrooke (GAUS), Département de Génie Mécanique, \\ Université de Sherbrooke, 2500 boul. de l'Université, Sherbrooke, Québec JIK 2RI, Canada
}

Camille Perrot $^{\text {b) }}$ and Vincent Monchiet

Université Paris-Est, Laboratoire Modélisation et Simulation Multi Echelle, MSME UMR 8208 CNRS,

5 bd Descartes, 77454 Marne-la-Vallée, France

\begin{abstract}
Raymond Panneton
Groupe d'Acoustique de l'Université de Sherbrooke (GAUS), Département de Génie Mécanique, Université de Sherbrooke, 2500 boul. de l'Université, Sherbrooke, Québec J1K 2RI, Canada
\end{abstract}

(Received 4 March 2016; revised 2 June 2017; accepted 8 June 2017; published online 26 June 2017)

\begin{abstract}
The main purpose of this article is to present, within a unified framework, a technique based on numerical homogenization, to model the acoustical properties of real fibrous media from their geometrical characteristics and to compare numerical results with experimental data. The authors introduce a reconstruction procedure for a random fibrous medium and use it as a basis for the computation of its geometrical, transport, and sound absorbing properties. The previously ad hoc "fiber anisotropies" and "volume weighted average radii," used to describe the experimental data on microstructure, are here measured using scanning electron microscopy. The authors show that these parameters, in conjunction with the bulk porosity, contribute to a precise description of the acoustical characteristics of fibrous absorbents. They also lead to an accurate prediction of transport parameters which can be used to predict acoustical properties. The computed values of the permeability and frequency-dependent sound absorption coefficient are successfully compared with permeability and impedance-tube measurements. The authors' results indicate the important effect of fiber orientation on flow properties associated with the different physical properties of fibrous materials. A direct link is provided between three-dimensional microstructure and the sound absorbing properties of non-woven fibrous materials, without the need for any empirical formulae or fitting parameters. (C) 2017 Acoustical Society of America. [http://dx.doi.org/10.1121/1.4989373]
\end{abstract}

$[\mathrm{KVH}]$

Pages: $4768-4780$

\section{INTRODUCTION}

Air-saturated fibrous materials are used in filtration and in thermal and sound insulation. These systems usually exhibit interesting sound absorbing properties and are, in some respects, widely used in the transportation and building industries. While several studies have devoted considerable attention to the description of the acoustical characteristics of fibrous absorbents, ${ }^{1-4}$ our understanding of the basic relationships between the structure and the acoustical properties of these fibrous webs is still to benefit from a better understanding. It would be particularly valuable to have a manageable set of geometrical parameters of the three-dimensional (3D) fiber web morphology, in terms of which the transport (e.g., static viscous $k_{0}$ and thermal $k_{0}^{\prime}$ permeabilities, viscous characteristic length $\Lambda$, high frequency tortuosity $\alpha_{\infty}$ ) and acoustical properties of non-woven fibrous media could be described. Obvious candidates for these parameters are the fiber diameters and the angular orientation distributions of fibers, as these parameters control most of the transport properties of non-woven fibrous materials, ${ }^{5}$ and therefore play a

\footnotetext{
a) Also at Université Paris-Est, Laboratoire Modélisation et Simulation Multi Echelle, MSME UMR 8208 CNRS, 5 bd Descartes, 77454 Marne-laVallée, France.

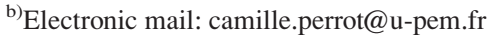

central role in the description of their sound absorbing characteristics. ${ }^{6}$

In this work, we introduce a simple reconstruction procedure of a random fibrous medium. The reconstructed fibrous medium is used as a basis for the computation of its geometrical, transport, and sound absorbing properties. In discussing a range of experimental data on the microstructure of non-woven fibrous materials involving x-ray micro computed tomography and microscopy analysis, previous works had relied on ad hoc "fiber anisotropies", "volume weighted average radius." ${ }^{9}$ Here it is shown that, in conjunction with the bulk porosity, these parameters:

(1) Contribute to a precise description of the acoustical characteristics of fibrous absorbents. In the present work, both parameters are implicitly measured from scanning electron microscope (SEM) images on two orthogonal cross-sections,

(2) lead to an accurate prediction of transport parameters which can be used to predict acoustical properties. The fiber morphology is linked to non-acoustical properties which control the acoustical behavior of these types of media (e.g., $\left.k_{0}, k_{0}^{\prime}, \Lambda, \alpha_{\infty}\right)$, and

(3) provide a direct link between 3D microstructure and sound absorbing properties of non-woven fibrous materials without the need for any empirical formulae or fitting parameters. 
In the present paper, we focus on $3 \mathrm{D}$ reconstruction. Indeed, the choice of a 3D structure allowed us to study the effect of fiber orientation on the classical transport properties, namely permeability, diffusion, and potential flow. The mathematical modeling of the classical transport properties is also a means to precisely describe the sound absorbing properties.

We developed an approach, inspired by a general methodology proposed by Adler and Thovert, ${ }^{10}$ but dedicated to fibrous media and taking account of their specific features. Our methodology consists of three steps. First, the salient geometric properties of a fibrous sample are measured. The angular orientation distributions of a fibrous material made of two different populations of fibers are characterized by SEM images from horizontal and vertical cross-sections of a slab of porous sample. Second, models of synthetic fibrous media are generated with the same statistical properties. Finally, the transport properties are obtained by integrating the relevant local partial differential equations. These transport parameters are used as input parameters for the determination of the frequency-dependent acoustical properties of the synthetic fibrous medium, which is treated as a homogeneous equivalent-fluid. The acoustics of air-filled fibrous media can be described within an equivalent-fluid approach through physics-based models. These models account for the inertial and viscous effects using an effective density $\rho_{\text {eq }}(\omega),{ }^{11}$ and for the thermal effects via an effective bulk modulus $K_{\text {eq }}(\omega) .^{12}$

Section II describes the measurement procedure for determination of geometrical properties. The geometrical properties provide input to our procedure for the generation of synthetic 3D fibrous samples. Then, an original reconstruction algorithm devised to precisely mimic the geometry of non-woven fiber webs is presented. Section III describes the numerical results for the computed macroscopic properties and their comparison with experimental data. Predictions relating to the macroscopic parameters, including the permeability and the sound absorption coefficient are then compared with measurements. The effect of the orientation of the fibers is also studied. Our results are discussed in Sec. IV. Finally, Sec. $\mathrm{V}$ is devoted to concluding remarks and suggestions for future research directions.

\section{NUMERICAL METHODS}

\section{A. Generation of random fibrous materials}

Here we describe how Protec-Style sustainable fibrous media (produced by Protec-Style, Quebec, Canada) are generated computationally. The non-woven fibrous materials used in this study are disordered structures of fibers randomly distributed in a 3D space and assumed to merge at the crossovers. We considered the fibers to be circular cylinders, with uniform fiber diameters. The geometrical characteristics of the cylinders in the generated fiber-webs were modeled using the experimentally determined fiber diameters and proportion of each type of fiber [natural fibers (fiber type $a$ ) and petro-sourced fibers (fiber type $b$ ) used as a thermal binder]. All the model structures were generated for a given porosity $(\phi=0.962 \pm 0.004)$, which corresponds to the measured porosity. ${ }^{13}$ The same generating protocol was followed for all fiber-webs. This approach enables us to capture the effect of the angular orientation at constant porosity or the effect of porosity at constant angular orientation.

Model random fibrous materials were generated based on the geometrical characteristics of a real non-woven fibrous sample made of $70 \%$ wt. of milkweed Asclepias (natural hollow fibers) and $30 \%$ wt. of bicomponent fibers comprised of two polymers within the same filament (PP and PE). The fibers are mechanically mixed and carded into webs which are stacked and thermobonded. The internal and external diameters of hollow fibers were estimated from SEM images. A S-3000N SEM (Hitachi, Tokyo, Japan) was used to acquire 28 images of horizontal ( $x y$-plane) and 28 images of vertical (orthogonal to the $x y$-plane) cross-sections, at different locations of the non-woven, among which 3601 and 3322 fiber orientation angles, respectively, were manually measured (see Appendix A). The experimental data were then converted into continuous probability density functions using uniform and normal estimates. The experimental histograms and the resulting probability density functions are shown in Fig. 1. These two types of cross-section are characterized by different behaviors of their angular orientations. The probability density function corresponding to the SEM images taken along the horizontal plane is uniform, whereas the angular orientation of the fibers corresponding to SEM images taken along the vertical plane is characterized by a mean orientation angle $\mu_{\theta}=89.73^{\circ}$ and a standard deviation $\sigma_{\theta}=13.63^{\circ}$. The angles of the fibers in the vertical plane were modeled using a normal distribution after the experimentally determined histograms. The statistical properties of the experimentally determined geometrical characteristics from SEM images are summarized in Table I. The typical length of natural fibers is 2 to $4 \mathrm{~cm}$, whereas the petrosourced fibers are much longer.

The reconstruction of a $3 \mathrm{D}$ random fiber web representative of the transport properties of real fibrous materials begins with the construction of a geometrical model preserving the measured porosity. A simplified model should be efficient enough to minimize computational cost and representative enough to capture the main geometrical features which could represent the transports phenomena. When the size of the box in which the cylindrical fibers are introduced is small, the solid volume for each new cylinder has a large effect on the calculated porosity, whereas the effect is small for a large box size. One might therefore expect the size of the Representative Elementary Volume (REV) to be such that the porosity of the reconstructed fiber webs reproduces the measured value. To quantify the size of the box as a function of porosity, we developed a semi-analytical model in which we could estimate the required number of fibers such that the porosity in the model is very close to the measured one.

Computations of the transport properties were made on a Periodic Unit Cell (PUC) as the REV. The PUC is a cubic box with dimension $L^{(i)}$ for iteration $i$. We recall that the indices $a$ and $b$ account for two different fiber types, i.e., Asclepias and bicomponent (synthetic) fibers, respectively. By determining the total fibers volumes $V_{a}^{(i)}$ 

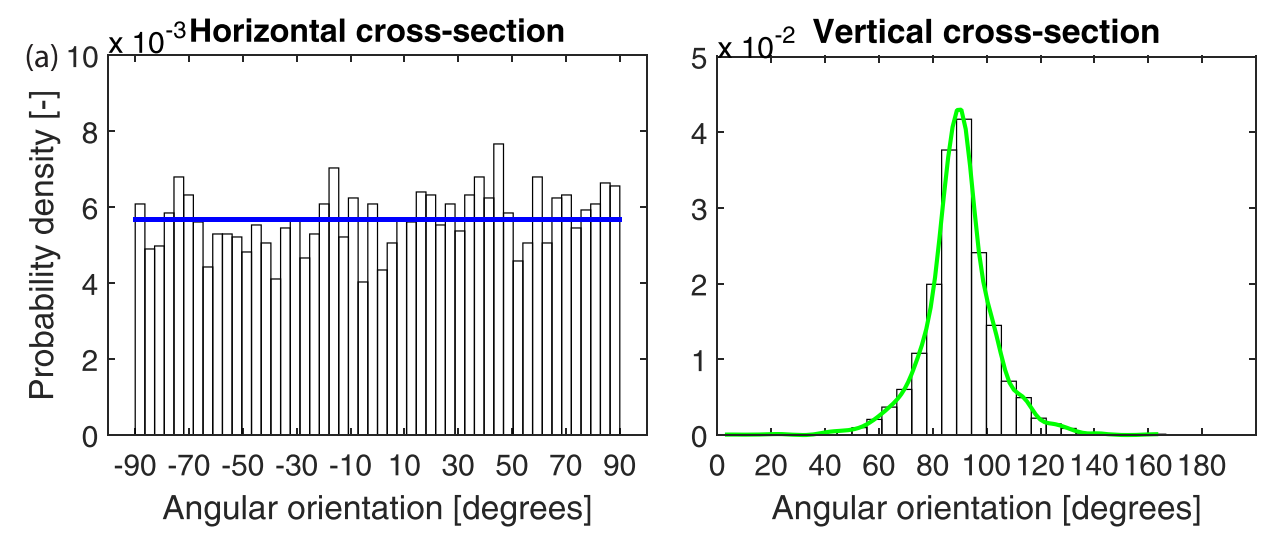

FIG. 1. (Color online) (a) The fiber orientation distributions as experimentally determined by SEM images. Histograms were converted into probability density functions. (b) SEM images corresponding to horizontal ( $x-y$ plane) and vertical (orthogonal to $x-y$ plane) cross-sections at a porosity $\phi_{h}=0.962 \pm 0.004$. Blue segments characterize the lengths and orientations of the fibers, as manually measured.

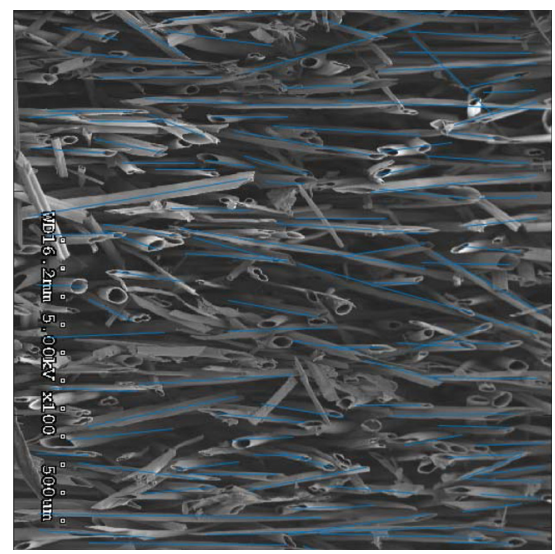

and $V_{b}^{(i)}$ inside the elementary volume $(i)$, it is possible to compute the required number of fibers $N_{a}^{(i)}$ and $N_{b}^{(i)}$ inside the cubic box in such a way that the porosity value will converge. In our case, the values of $V_{a}^{(i)}$ and $V_{b}^{(i)}$ are defined analytically from the targeted (final) porosity $\phi$, the cubic box volume $L^{(i) 3}$, the fiber radii $r_{a}$ and $r_{b}$, the wall thickness $t$ of the hollow Asclepia fiber, and the ratio between bicomponent and Asclepias fibers $L_{b} / L_{a}$ according to (Appendix B)

TABLE I. Statistical properties of the experimentally determined geometrical characteristics of fibers from SEM images.

\begin{tabular}{|c|c|c|}
\hline & $\begin{array}{l}\text { Asclepias } \\
\text { fibers (a) }\end{array}$ & $\begin{array}{l}\text { Bicomponent } \\
\text { fibers (b) }\end{array}$ \\
\hline \multicolumn{3}{|l|}{ Fiber lengths } \\
\hline Number of measured fiber segments & 1959 & 1140 \\
\hline Fiber segments cumulated length $(L), \mu \mathrm{m}$ & 467840 & 173820 \\
\hline \multicolumn{3}{|l|}{ Fiber diameters } \\
\hline Number of measured fibers & 744 & 184 \\
\hline Mean diameter $(2 r), \mu \mathrm{m}$ & 25 & 14.7 \\
\hline Standard deviation $\left(\sigma_{d}\right), \mu \mathrm{m}$ & 6.6 & 1.7 \\
\hline \multicolumn{3}{|l|}{ Fiber wall thicknesses } \\
\hline Number of measured fibers & 119 & - \\
\hline Mean thickness $(t), \mu \mathrm{m}$ & 1.6 & - \\
\hline Standard deviation $\left(\sigma_{t}\right), \mu \mathrm{m}$ & 0.4 & - \\
\hline \multicolumn{3}{|l|}{ Fiber vertical orientation angles } \\
\hline Number of measured fibers & \multicolumn{2}{|r|}{3322} \\
\hline Mean angle $\left(m_{q}\right)$, degree & \multicolumn{2}{|r|}{89.73} \\
\hline Standard deviation $\left(\sigma_{q}\right)$, degree & \multicolumn{2}{|r|}{13.63} \\
\hline Min. angle $\left(q_{\min }\right)$, degree & \multicolumn{2}{|r|}{0.00} \\
\hline Max. angle $\left(q_{\max }\right)$, degree & \multicolumn{2}{|r|}{166.53} \\
\hline
\end{tabular}

$$
\begin{gathered}
V_{a}^{(i)}=\frac{(1-\phi) L^{(i) 3}}{1+\frac{L_{b}}{L_{a}} \times \frac{r_{b}^{2}}{r_{a}^{2}-\left(r_{a}-t\right)^{2}}}, \\
V_{b}^{(i)}=\frac{L_{b}}{L_{a}} \times \frac{r_{b}^{2}}{r_{a}^{2}-\left(r_{a}-t\right)^{2}} \times V_{a}^{(i)} .
\end{gathered}
$$

Then, the iterative determination of the number of fibers $N_{a}^{(i)}$ and $N_{b}^{(i)}$ starts by considering a 3D and two-phase (fiber/air) fibrous material [whose internal structure is shown in Fig. 1(b)]. Each iteration consists of three sequential steps: (i) Randomly locate the fiber core, $c_{j}$, a position $\left(x_{j}, y_{j}, z_{j}\right)$ inside the elementary volume according to a uniform distribution. (ii) Assign a random number pair to the orientation angles $\left(\varphi_{j}, \theta_{j}\right)$ for each fiber core to define the fiber orientation. For the present study, the horizontal orientation angle $\varphi_{j}$ can be any value within $\left[0,2 \pi\left[\right.\right.$. Values of the vertical angles $\left(\theta_{j}\right)$ are determined by the experimentally determined distribution. Here, we used a normal probability density function. (iii) Calculate the fiber length $l_{j}$ inside the square of side $L^{(i)}$, from which the elementary solid volumes are derived for each type of fiber (nested loops). As the sums of these elementary solid volumes asymptotically approach $V_{a}^{(i)}$ and $V_{b}^{(i)}$, the current porosity $\phi^{(i)}$ approaches $\phi$. The number of iterations $i$ defines the number of fibers $N_{a}^{(i)}$ and $N_{b}^{(i)}$. This procedure essentially allows iterative alteration of the cubic box dimension $L^{(i)}$ until porosity is converged. $L^{(i)}$ was chosen as a variable in the PUC modeling to ensure that the porosity of the PUC corresponds to the experimental porosity with a controlled fluctuation. The implemented algorithm is depicted in Fig. 2. Applying it for 1000 iterations corresponding to each 


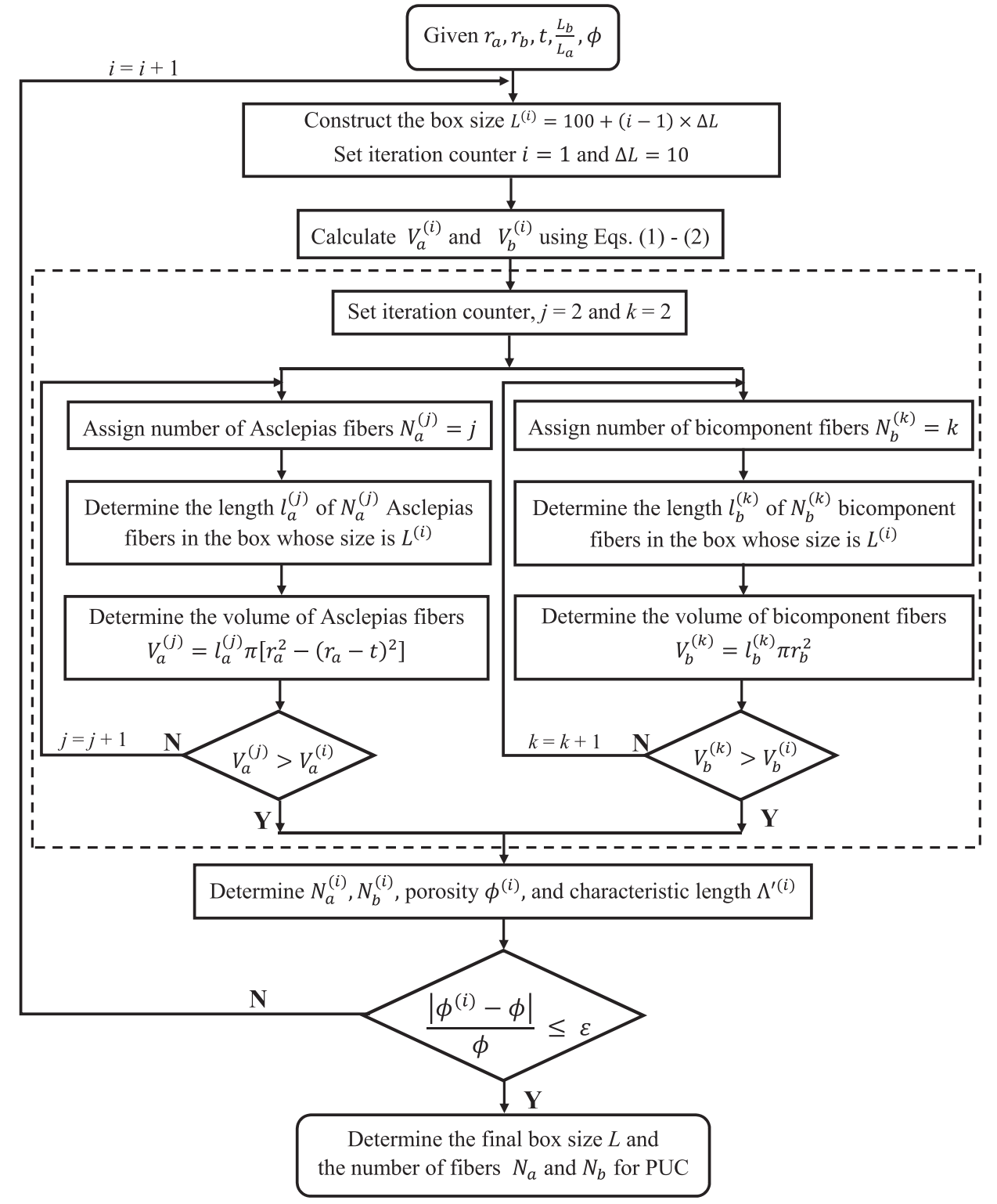

FIG. 2. The iterative procedure to calculate the cubic box dimension and the number of fibers for the given fibrous material geometrical characteristics and porosity. box size resulted in a minimum mean value of the final box size $L$ equal to $400 \mu \mathrm{m}$, to obtain a relative porosity difference between the experimental and calculated values of less than $\varepsilon=0.1 \%$. Thus, the fibers are long when compared to the size of the PUC. The program realization of the box-size-algorithm was implemented as a parallel code in MATLAB language. The total simulation time was $\sim 163 \mathrm{~s}$ on $3.47 \mathrm{GHz} / 8$ processor cores (160 GB RAM). Figure 3 shows the 3D view [Fig. 3(a)] and front view [Fig. 3(b)] of a generated fiber packing at a porosity $\phi_{h}=0.962$. In these illustrations, the gray lines define the Asclepias fibers, whereas the black lines represent the bicomponent fibers. As the cubic box dimension iteratively increases, the porosity asymptotically approaches the targeted (experimental) porosity $\phi$ while reducing the standard deviation; Fig. 3(c).

\section{B. Simulation of transport properties}

As in our previous paper, ${ }^{14}$ Stokes flow, potential flow, and heat conduction in the generated fiber webs were simulated using a finite element (FEM) scheme. ${ }^{14}$ The transverse permeability $k_{0}$ for the Stokes flow, also called the throughplane permeability, was obtained by averaging the velocity components in the direction of the flow (as defined for instance in Sec. II C of Ref. 14). Similarly, the "thermal permeability" $k_{0}^{\prime}$ satisfies the mean value of the "scaled concentration field" $u_{p}(\vec{r})$, where $u_{p}(\vec{r})$ solves $\Delta u_{p}(\vec{r})=-1$ in the fluid filling the space $V_{1}$ between the fibers and the walls of the fibers, and $u_{p}(\vec{r})=0$ on the walls of the fibers $\partial V{ }^{15}$ $k_{0}^{\prime}$ is indeed equal to $\left\langle u_{p}(\vec{r})\right\rangle$, where \langle\rangle denotes a fluid-phase ensemble average of volume fraction $\phi$ [see Eqs. (2.10), (2.11), and (2.13) of Ref. 16 for a derivation of this result]. In the context of diffusion-controlled processes, $u_{p}(\vec{r})$ is a scaled concentration field and is defined as the rate of diffusion of the diffusing species divided by the diffusion coefficient of the reactive particles. For thermal diffusion, $u_{p}(\vec{r})$ is a "temperature field", defined as the ratio of the time derivative of the acoustic pressure over the thermal conductivity of the solid frame. It is worthwhile noticing that $\Delta u_{p}$ is dimensionless, therefore $u_{p}(\vec{r})$ and $k_{0}^{\prime}$ have the dimension of a surface. The viscous characteristic length $\Lambda$ and the tortuosity 
(a)

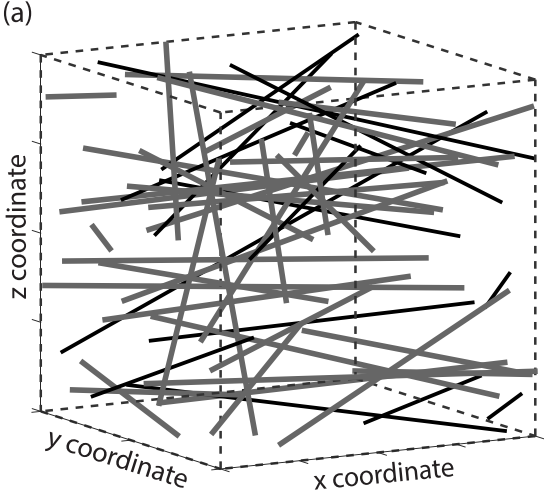

(b)

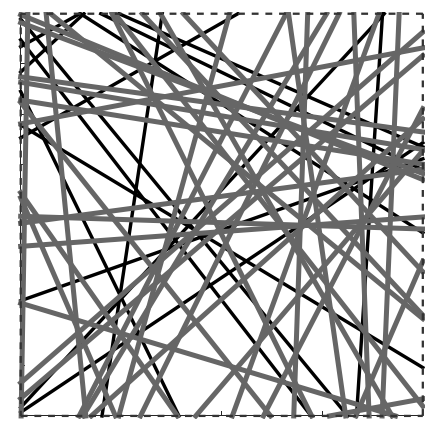

(c)

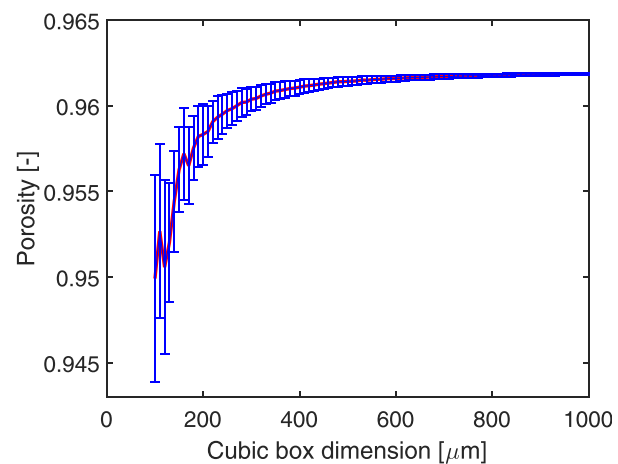

FIG. 3. (Color online) 3D reconstruction of a random fibrous medium. (a) Illustration of a typical model made of two type of fibers with different fiber diameters. The angular orientation of fibers statistically reproduces measurements obtained from SEM images. (b) The corresponding two-dimensional view of a computer-generated fiber web illustrates the impression obtained by looking at a small piece of such a fibrous material $(L=0.4 \mathrm{~mm})$. (c) The developed approach for generating the microstructure displays a porosity convergence as a function of the cubic box dimension towards the measured value. The mean porosity values (in red) are accompanied by a standard deviation (in blue) corresponding to 1000 realizations.

$\alpha_{\infty}$ were calculated from their definitions [see Ref. 11, Eqs. (2.9) and (2.17)] as

$$
\begin{gathered}
\alpha_{\infty}=\frac{\phi A\left|\Psi_{L}\right|^{2}}{L \int\left|\vec{u}_{p}(\vec{r})\right|^{2} d V_{1}}, \\
\Lambda=2 \frac{\int\left|\vec{u}_{p}(\vec{r})\right|^{2} d V_{1}}{\int\left|\vec{u}_{p}(\vec{r})\right|^{2} d S},
\end{gathered}
$$

where $\vec{u}_{p}(\vec{r})$ is the microscopic potential-flow solution, $L$ is the thickness of the porous element and $A$ is its lateral area, $d V_{1}$ denotes an integration over the pore volume, and $d S$ denotes an integration over the pore-solid surface. Here, $\Psi(z=0)=0$ and $\Psi(z=L)=\Psi_{L}$ (two constants) are two boundary conditions associated with a potential difference applied between the ends of the sample. In Eqs. (3) and (4), $\vec{u}_{p}(\vec{r})=-\vec{\nabla} \Psi$ is determined by the solution of the Laplace equation $\Delta \Psi=0$. At the pore walls inside the medium the normal components of the potential $\Psi$ vanish: $\vec{n} \cdot \vec{\nabla} \Psi=0$ is usually referred as the Neumann condition, where $\vec{n}$ is the outward unit vector normal to the boundary $\partial V$. It can be demonstrated that the magnitude of $\alpha_{\infty}$ is greater than or equal to one and the equality takes place only if the pore space is a bundle of straight channels. In this numerical study, we considered only solid fibers and thus neglected the flow inside the hollow fibers. We generated a tetrahedralization of the pore space. This tetrahedralization was then used to create a finite element (FE) mesh for the COMSOL Multiphysics ${ }^{17}$ Stokes, Poisson, and Laplace solvers. For all the available numerical schemes the flow was evaluated in direction $z$. Simulations were carried out with periodic boundary conditions (see Ref. 14 for more details). This means that the solution fields are constrained to be equal on opposite faces of the cubic box, with a geometry which is not periodic. This is a simple means of increasing convergence of the solution with respect to the size of the cubic box. ${ }^{18}$ Figure 4 shows the solution fields evaluated by FEM numerical solutions for flows in the $z$ direction and diffusion. Note that, visually, the fluid-flow paths are clearly more concentrated, and follow a more tortuous path, than do the potential velocity paths. This is due mainly to the no-slip boundary condition. Moreover, as already mentioned by Boutin and Geindreau ${ }^{19}$ the heat diffusion field is independent of the orientation of the fibers. (It can be viewed as the superposition of the flow field in the three main directions.) The typical simulation times for Stokes flow, potential flow, and heat diffusion problems of transport are, respectively $442 \pm 47 \mathrm{~s}, 14 \pm 2 \mathrm{~s}$, and $15 \pm 2 \mathrm{~s}$ (using a computer with a $3.40 \mathrm{GHz}$ processor core and $24 \mathrm{~GB}$ RAM).

\section{Estimation of acoustic properties}

Based on the approximate formulae developed by Johnson et al. ${ }^{11}$ and Lafarge et al., ${ }^{12}$ the effective density $\rho_{\text {eff }}(\omega)$ and the effective bulk modulus $K_{\text {eff }}(\omega)$ of the fluid phase can be evaluated as follows:

$$
\begin{aligned}
& \rho_{\mathrm{eff}}(\omega)=\rho_{o} \alpha_{\infty}\left[1+\frac{1}{\varpi} f(\varpi)\right], \\
& \frac{1}{K_{\mathrm{eff}}(\omega)}=\frac{1}{K_{a}}\left\{\gamma-(\gamma-1)\left[1+\frac{1}{j \varpi^{\prime}} f^{\prime}\left(\varpi^{\prime}\right)\right]^{-1}\right\},
\end{aligned}
$$

where $\rho_{0}$ is the air density at rest, $K_{a}$ is the adiabatic bulk modulus of air, and $\gamma$ is its specific heat ratio. The quantities $\varpi$ and $\varpi^{\prime}$ are dimensionless frequencies given by the following expressions:

$$
\begin{aligned}
& \varpi=\frac{\omega}{\nu} \frac{k_{0} \alpha_{\infty}}{\phi}, \\
& \varpi^{\prime}=\frac{\omega}{\nu^{\prime}} \frac{k_{0}^{\prime}}{\phi}
\end{aligned}
$$

with $\nu^{\prime}=\nu / \operatorname{Pr}, \nu$ being the kinematic viscosity and $\operatorname{Pr}$ is the Prandtl number ( $\cong 0.71$ for air). $f(\varpi)$ and $f^{\prime}\left(\varpi^{\prime}\right)$ are shape functions defined by

$$
f(\varpi)=\sqrt{1+\frac{M}{2} j \varpi}
$$



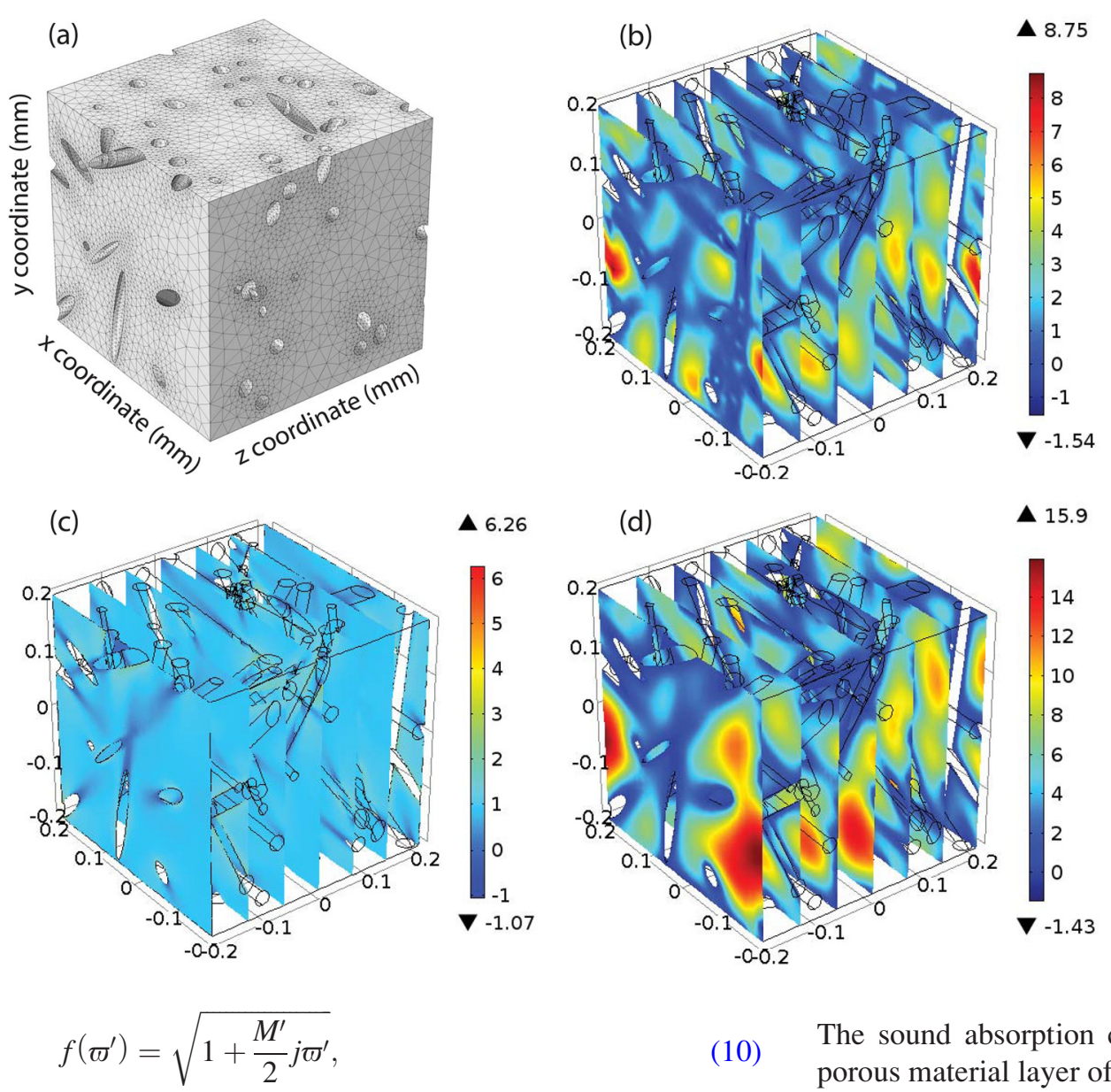

$M$ and $M^{\prime}$ are referred to as dimensionless shape factors determined from

$$
\begin{aligned}
& M=\frac{8 k_{0} \alpha_{\infty}}{\Lambda^{2} \phi}, \\
& M^{\prime}=\frac{8 k_{0}^{\prime}}{\Lambda^{\prime 2} \phi} .
\end{aligned}
$$

$\rho_{\text {eq }}(\omega)=\rho_{\text {eff }}(\omega) / \phi$ and $K_{\text {eq }}(\omega)=K_{\text {eff }}(\omega) / \phi$ are the equivalent density and bulk modulus of the so-called rigid-frame equivalent-fluid medium. The thermal characteristic length ${ }^{20}$ $\Lambda^{\prime}$ introduced in Eq. (12) is similar to Eq. (4), but the volume and area elements are not weighted by the microscopic potential-flow solution. The characteristic length $\Lambda^{\prime}$ is a purely geometrical parameter equal to twice the volume-topore-surface ratio which can be computed directly from the mesh of the FEM scheme. Assuming plane wave solutions varying as $\exp \left[j\left(\omega t-q_{\mathrm{eq}}(\omega) x\right)\right]$, where $q_{\mathrm{eq}}(\omega)$ represents the wave number of the equivalent fluid medium, $\rho_{\text {eq }}(\omega)$ and $K_{\text {eq }}(\omega)$ can be used to calculate the wave number and the characteristic impedance of the equivalent fluid medium with

$$
\begin{aligned}
& q_{\mathrm{eq}}=\omega \sqrt{\frac{\rho_{\mathrm{eq}}(\omega)}{K_{\mathrm{eq}}(\omega)}}, \\
& Z_{c \mathrm{eq}}=\sqrt{\rho_{\mathrm{eq}}(\omega) K_{\mathrm{eq}}(\omega)} .
\end{aligned}
$$

The sound absorption coefficient at normal incidence of a porous material layer of thickness $d$ backed by a rigid wall is evaluated by

$$
\alpha=1-\left|\frac{Z_{n}-1}{Z_{n}+1}\right|^{2},
$$

with

$$
Z_{n}=-j \frac{Z_{c \mathrm{eq}}}{Z_{0}} \cot \left(q_{\mathrm{eq}} d\right),
$$

the effective normal impedance on the free face of the excited material, where $Z_{0}$ is the characteristic impedance of ambient air. Then, when the sample is on an anechoic termination, the normal incidence transmission coefficient $\tau_{\infty}$ is determined from the equivalent wave number and the equivalent characteristic impedance of the acoustical material as

$$
\tau_{\infty}=\frac{2 e^{j q_{0} d}}{2 \cos \left(q_{\mathrm{eq}} d\right)+j \frac{Z_{c \mathrm{eq}}}{Z_{0}} \sin \left(q_{\mathrm{eq}} d\right)+j \frac{Z_{0}}{Z_{c \mathrm{eq}}} \sin \left(q_{\mathrm{eq}} d\right)}
$$

Finally, the normal incidence sound transmission loss (TL) is obtained from

$$
\mathrm{TL}=-20 \log \left(\left|\tau_{\infty}\right|\right)
$$

When the frame of the porous material is assumed limp (i.e., flexible), the effective density is modified as follows: ${ }^{21}$ 
TABLE II. Macroscopic parameters: measurements and computational results.

\begin{tabular}{|c|c|c|c|c|c|c|c|}
\hline & \multicolumn{2}{|c|}{$\phi(-)$} & \multirow[b]{2}{*}{$\Lambda^{\prime}(\mu \mathrm{m})$} & \multirow[b]{2}{*}{$\alpha_{\infty}(-)$} & \multirow[b]{2}{*}{$\Lambda(\mu \mathrm{m})$} & \multirow[b]{2}{*}{$k_{0}\left(\times 10^{-11} \mathrm{~m}^{2}\right)$} & \multirow[b]{2}{*}{$k_{0}^{\prime}\left(\times 10^{-11} \mathrm{~m}^{2}\right)$} \\
\hline & hollow fibers, $\phi_{h}$ & solid fibers, $\phi_{s}$ & & & & & \\
\hline Measurements & $0.962 \pm 0.004$ & & & & & $19.571 \pm 1.184$ & \\
\hline Computations & $0.963 \pm 0.003$ & $0.900 \pm 0.003$ & $103 \pm 4$ & $1.054 \pm 0.003$ & $51 \pm 2$ & $19.247 \pm 0.892$ & $61.838 \pm 5.848$ \\
\hline
\end{tabular}

$$
\rho_{\mathrm{eq}}^{\prime}(\omega)=\frac{\rho_{\mathrm{eq}}(\omega) m-\rho_{0}^{2}}{m+\rho_{\mathrm{eq}}(\omega)-2 \rho_{0}},
$$

where $m=\rho_{1}+\phi \rho_{0}$ is the total apparent mass of the equivalent fluid limp medium, and $\rho_{1}$ is the in vacuo bulk density of the medium.

Therefore, an estimation of the acoustic properties of random fibrous media can be obtained on the basis of six macroscopic parameters $\left(\phi, \Lambda^{\prime}, k_{0}, k_{0}^{\prime}, \alpha_{\infty}, \Lambda\right)$ determined from the numerical simulation of three different transport phenomena (viscous fluid flow, perfect fluid flow, heat diffusion) for rigid fiber packings of thickness $d$.

\section{RESULTS}

In Table II, we present the computed values of transport parameters for the Asclepias/bicomponent fiber assembly having a measured porosity of $\phi=0.962 \pm 0.004$, an in vacuo bulk density of $\rho_{1}=32.30 \pm 0.07 \mathrm{~kg} \mathrm{~m}^{-3}$ (three samples were measured to create the statistics for these values), and the fiber orientation distributions shown in Fig. 1. Practically, we employed the proposed numerical approach to generate random webs of solid fibers. Because we determined the transport properties for the packing of solid cylinders instead of hollow cylinders, the porosity of the reconstructed fiber webs is equal to $\phi_{s}=0.900 \pm 0.003$. This value corresponds to the value calculated for geometrical solid cylinders of identical lengths and outer diameters. The simulated and measured permeability ${ }^{22}$ values are very close to each other: $19.571 \pm 1.184 \times 10^{-11} \mathrm{~m}^{2}$ for the experimental samples and $19.247 \pm 0.892 \times 10^{-11} \mathrm{~m}^{2}$ for the reconstructed fiber webs. The $1.66 \%$ difference between the viscous permeabilities for the fibrous samples under study and for the simulated fiber webs indicates that the fibrous webs capture the essential physics of the viscous dissipation effects. Ten samples were used to create the statistics for the macroscopic properties of the simulated fiber webs. We also present in Fig. 5(a) a comparison between the experimental sound absorption at normal incidence and the estimate combining the FE results for the transport parameters (Sec. II B) and the semi-phenomenological analytical models (rigid and limp) for the acoustic properties (Sec. IIC). Except around the resonance frequency, we see that both rigid and limp estimates agree with the experimentally measured sound absorbing coefficient: 0.69 for the limp model, 0.61 for the rigid model, and $0.57 \pm 0.06$ for the measured sound absorption at $1600 \mathrm{~Hz}$, a frequency corresponding to the largest relative differences in the rest of the frequency spectrum. This strongly supports the idea that the reconstructed random fibrous materials capture the essential transport parameters-because our sound absorption prediction contains transport coefficients that depend on the arrangement of the fibers. It is noteworthy that using the doubleporosity theory results in a sound absorption increasing slightly at high frequencies (not shown here). This minor effect of fiber hollowness corresponds to a low interscale coupling. We notice that all of the proposed models (rigid, ${ }^{11,12}$ limp,${ }^{21}$ elastic, ${ }^{23}$ ) predict an overall sound absorption in agreement with measurements. With the elastic model, the sound absorption is almost always in accord with measurements. The maximum relative difference is observed around the resonance frequency where the measured sound absorption is about $0.20 \pm 0.04$ at $1000 \mathrm{~Hz}$, whereas that for the elastic model is equal to 0.30 at the same frequency. This relative difference must be balanced by the fact that the sound absorption is very sensitive to mounting conditions around the resonance frequency. Thus, the small differences between experimental and modeling data, such as permeability (Table II) or sound absorption [Fig. 5(a)], on the one hand for a stack of solid fibers and on the other hand for real samples containing hollow fibers indicate that a simplified model of the reconstructed fibrous geometry can be used, preserving the outer dimensions of the fibers in a FE model without any inner porosity meshing.

In the rigid case, the model used was the JohnsonLafarge one presented in Sec. IIC, with the computed
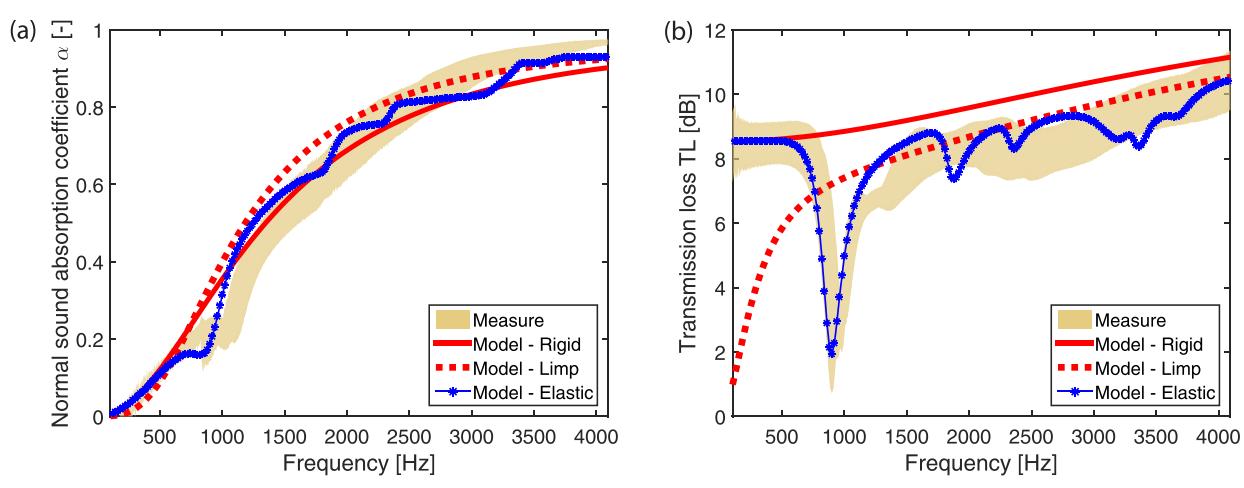

FIG. 5. (Color online) A comparison between measurements and prediction: (a) normal incidence sound absorption coefficient, (b) TL. The sample thickness $d$ is equal to $14.30 \pm 0.89 \mathrm{~mm}$. 
parameters given in Table II including $\phi_{s}=0.900$. Indeed, to meet double porosity model requirements, the microporous medium must be sufficiently permeable to acoustical waves, and the micropore characteristic size must be greater than $10 \mu \mathrm{m} .{ }^{24}$ As the fiber inner diameter is close to that limit with a value of about $22 \mu \mathrm{m}$, the acoustical wave should not penetrate much into the fibers' internal porosity. We also used the limp model in which the effective density of the rigid-frame equivalent fluid medium is corrected as per Eq. (19). In the elastic case, an axisymmetrical FE poroelastic model was used. In that model, the same $\rho_{\text {eq }}(\omega)$ and $K_{\text {eq }}(\omega)$ as described in Sec. IIC were used for the fluid phase. To identify the elastic properties of the fibrous material, we therefore used an axisymmetrical FE model with a Biot formulation in which we could replicate the mounting conditions of the samples in the impedance tube while explicitly representing the transport properties that were directly computed previously. This exploration is however reported in Appendix $\mathrm{C}$ since the main objective of this work is to predict acoustic properties while avoiding any fitting parameter; whereas the latter FE model does not allow for the direct micro-/macro numerical computation of the elastic parameters.

The Johnson et al. ${ }^{11}$ and Lafarge et al. ${ }^{12}$ models assume a porous material having a rigid frame. In this situation, the experimentally measured effective density and effective bulk modulus could be used to quantify $\alpha_{\infty}, \Lambda, \Lambda^{\prime}$, and $k_{0}^{\prime}$ from analytical inversion, ${ }^{25,26}$ if one directly measures the porosity $\phi$ and the permeability $k_{0} \cdot{ }^{27}$ As rigid-frame behavior cannot be completely assumed here (see Fig. 5 and Appendix C), we used direct measurements of the permeability and the sound absorption of the fibrous material under study to validate this reconstruction approach.

To further validate the results, the simulated transport parameters were compared with the analytical values proposed for fibrous media by Allard and Champoux. ${ }^{2}$ In particular, we checked whether the thermal permeability $k_{0}^{\prime}$ and the viscous permeability were linked by $k_{0}^{\prime}=4 \times k_{0}$ [Eq. (46a) of Ref. 2]. In the case investigated the ratio $k_{0}^{\prime} / k_{0}$ is $3.21 \pm 0.14\left(\Omega_{\mathrm{zz}}=0.054\right)$, and we also verify that the ratio $k_{0}^{\prime} / k_{0}$ increases from $1.15 \pm 0.16$ at $\Omega_{\mathrm{zz}}=1$ to $3.14 \pm 0.15$ at
$\Omega_{\mathrm{zz}}=0$ (see the discussion on the effect of the orientation of fibers and Table III below). The remaining analytical estimates $\Lambda^{\prime}=2 \times \Lambda$ [Eq. (41) of Ref. 2] and $\alpha_{\infty}=1$ [Eq. (46b) of Ref. 2] are fully consistent with our numerical data (Table II). Although the numerical ratio $k_{0}^{\prime} / k_{0}$ is not equal to 4-presumably because $\phi_{s}=0.900 \pm 0.003$ (the equations are valid for very thin fibers) - the close match between the values of the transport coefficients in the numerical simulations and the analytical model, together with the experimental validations above, confirms that the reconstruction approach captures the essential physics of the visco-thermal losses in fibrous materials.

The main conclusion which can be drawn from the data presented in Table II and Fig. 5 is that the use of the generated random fibrous material reconstructed from the experimentally determined geometrical characteristics of single fibers, probability density functions for angular orientations, and porosity, allows an accurate estimate of the transport and the sound absorbing properties of the sample we analyzed in this study. Thus, the proposed approach gives a good estimate of the sound absorbing properties of random fibrous materials, using only information on the fibrous network geometry.

The numerical method developed here will now be used to simulate the effect of fiber orientation on the transport properties of a random fibrous material. The degree of alignment of fiber networks may be characterized using the second-order fiber orientation tensor $[\Omega]{ }^{30}$ Here, we adopt the approach of Advani and Tucker,${ }^{30}$ for which the full range of angular orientation states in fibrous materials is defined using a second-order fiber orientation tensor given by an integral over all directions of unit vectors $\vec{p}$ associated with each fiber axis

$$
[\Omega]=\Omega_{i j}=\int p_{i} p_{j} \psi(\vec{p}) d \vec{p}, \quad(i, j=1,2,3),
$$

where $\psi(\vec{p})=\psi(\varphi, \theta)$ denotes the probability distribution function for fiber orientation, and $\varphi$ and $\theta$ are the orientation angles of the fibers in the horizontal and vertical planes, respectively (Fig. 6). The components of $\vec{p}$ are related to $\varphi$ and $\theta$ such that

TABLE III. Macroscopic parameters determined by numerical simulation as a function of the statistically averaged angular orientation of fibers $\Omega_{z z}$.

\begin{tabular}{|c|c|c|c|c|c|c|}
\hline$\Omega_{z z}$ & $\phi(-)$ & $\Lambda^{\prime}(\mu \mathrm{m})$ & $\alpha_{\infty}(-)$ & $\Lambda(\mu \mathrm{m})$ & $k_{0}\left(\times 10^{-11} \mathrm{~m}^{2}\right)$ & $k_{0}^{\prime}\left(\times 10^{-11} \mathrm{~m}^{2}\right)$ \\
\hline 0 & $0.896 \pm 0.004$ & $101.0 \pm 4.6$ & $1.062 \pm 0.006$ & $48.7 \pm 2.5$ & $18.678 \pm 1.084$ & $58.735 \pm 5.323$ \\
\hline 0.054 & $0.900 \pm 0.003$ & $103.133 \pm 3.599$ & $1.054 \pm 0.003$ & $50.779 \pm 1.749$ & $19.247 \pm 0.892$ & $61.838 \pm 5.848$ \\
\hline 0.1 & $0.898 \pm 0.008$ & $102.3 \pm 6.9$ & $1.051 \pm 0.006$ & $51.4 \pm 3.7$ & $19.637 \pm 1.137$ & $59.335 \pm 3.612$ \\
\hline 0.2 & $0.900 \pm 0.003$ & $102.8 \pm 2.3$ & $1.043 \pm 0.003$ & $54.2 \pm 2.0$ & $20.742 \pm 0.927$ & $59.502 \pm 4.088$ \\
\hline 0.3 & $0.896 \pm 0.004$ & $100.8 \pm 3.5$ & $1.038 \pm 0.004$ & $56.4 \pm 2.9$ & $21.769 \pm 1.037$ & $60.113 \pm 3.894$ \\
\hline 0.4 & $0.894 \pm 0.004$ & $99.5 \pm 4.1$ & $1.030 \pm 0.003$ & $60.4 \pm 3.9$ & $23.153 \pm 1.695$ & $58.545 \pm 3.115$ \\
\hline 0.5 & $0.899 \pm 0.006$ & $101.8 \pm 3.6$ & $1.025 \pm 0.004$ & $64.4 \pm 2.9$ & $25.257 \pm 1.168$ & $59.211 \pm 3.442$ \\
\hline 0.6 & $0.895 \pm 0.005$ & $100.2 \pm 4.4$ & $1.019 \pm 0.004$ & $67.7 \pm 3.4$ & $25.864 \pm 1.429$ & $59.702 \pm 3.439$ \\
\hline 0.7 & $0.898 \pm 0.005$ & $102.1 \pm 5.2$ & $1.014 \pm 0.003$ & $73.1 \pm 5.4$ & $32.499 \pm 2.249$ & $60.175 \pm 4.647$ \\
\hline 0.8 & $0.897 \pm 0.004$ & $99.7 \pm 4.3$ & $1.006 \pm 0.001$ & $80.2 \pm 2.6$ & $39.817 \pm 2.716$ & $59.178 \pm 2.664$ \\
\hline 0.9 & $0.899 \pm 0.004$ & $103.1 \pm 4.2$ & $1.002 \pm 0.001$ & $89.9 \pm 4.4$ & $42.184 \pm 3.602$ & $61.660 \pm 4.535$ \\
\hline 1 & $0.887 \pm 0.003$ & $95.4 \pm 3.8$ & $1.000 \pm 0.000$ & $94.1 \pm 3.8$ & $53.892 \pm 6.779$ & $62.117 \pm 2.280$ \\
\hline
\end{tabular}




$$
\left\{\begin{array}{l}
p_{1}=\sin \theta \cos \varphi \\
p_{2}=\sin \theta \sin \varphi \\
p_{3}=\cos \theta
\end{array} \quad \text { and } \quad \int d \vec{p}=\int_{\varphi=0}^{2 \pi} \int_{\theta=0}^{\pi} \sin \theta d \theta d \varphi\right.
$$

Substituting Eq. (21) into Eq. (20) gives

$$
\Omega=\frac{1}{N_{f}} \sum_{i=1}^{N_{f}}\left[\begin{array}{ccc}
\sin ^{2} \theta^{(i)} \cos ^{2} \varphi^{(i)} & \sin ^{2} \theta^{(i)} \cos \varphi^{(i)} \sin \varphi^{(i)} & \sin \theta^{(i)} \cos \theta^{(i)} \cos \varphi^{(i)} \\
\sin ^{2} \theta^{(i)} \cos \varphi^{(i)} \sin \varphi^{(i)} & \sin ^{2} \theta^{(i)} \sin ^{2} \varphi^{(i)} & \sin \theta^{(i)} \cos \theta^{(i)} \sin \varphi^{(i)} \\
\sin \theta^{(i)} \cos \theta^{(i)} \cos \varphi^{(i)} & \sin \theta^{(i)} \cos \theta^{(i)} \sin \varphi^{(i)} & \cos ^{2} \theta^{(i)}
\end{array}\right]
$$

where $N_{f}=N_{a}+N_{b}$ is the total number of fibers; $\theta^{(i)}$ is the angle formed between the $i$ th fiber axis and the $z$ axis, and $\varphi^{(i)}$ is the angle formed between the projection of the $i$ th fiber on the $x$ - $y$ plane and the $x$ axis. Note that another definition of the fiber orientation tensor was proposed by Stylianopoulos et al., ${ }^{31}$ in which the fiber lengths $l_{i}$ and the total length of fibers $l_{\text {tot }}$ are also considered. The trace of $[\Omega]$ is always equal to 1 . For the isotropic case, $\Omega_{x x}=\Omega_{y y}=\Omega_{z z}=1 / 3$ for aligned networks, the value of the diagonal components is a measure of the fiber alignment in the coordinate directions. Off-diagonal components indicate a significant alignment in a direction other than a coordinate direction. For the current material, off-diagonal components are equal to zero. A central assumption underlying this property is that the fibers principal directions are superposed with the axes $\vec{e}_{1}, \vec{e}_{2}, \vec{e}_{3}$. According to Eq. (22), $[\Omega]$ takes on the values $\Omega_{x x}=\Omega_{y y}$ $=0.473$ and $\Omega_{z z}=0.054$ if $\varphi$ and $\theta$ adopt the fiber orientation distributions as experimentally determined (Fig. 1). These statistically averaged values of the angular orientation of the fibers are such that the resulting orientation tensor matched the experimental observation, Fig. 1(b), right. This result corresponds to a transversely isotropic fibrous configuration $\left(\Omega_{x x}=\Omega_{y y} \neq \Omega_{z z}\right.$ ) with fibers slightly inclined relative to the $x$-y plane $\left(\Omega_{z z}>0, \Omega_{z z} \ll 1\right)$. Figure $1(\mathrm{~b})$, right displays the angular orientation of fibers for which $\theta$ is, in general, different from $90^{\circ}$. Because the fibers are mechanically mixed and carded into webs, the fibers adopt an angular orientation that may cause a modification of the transport parameters. On

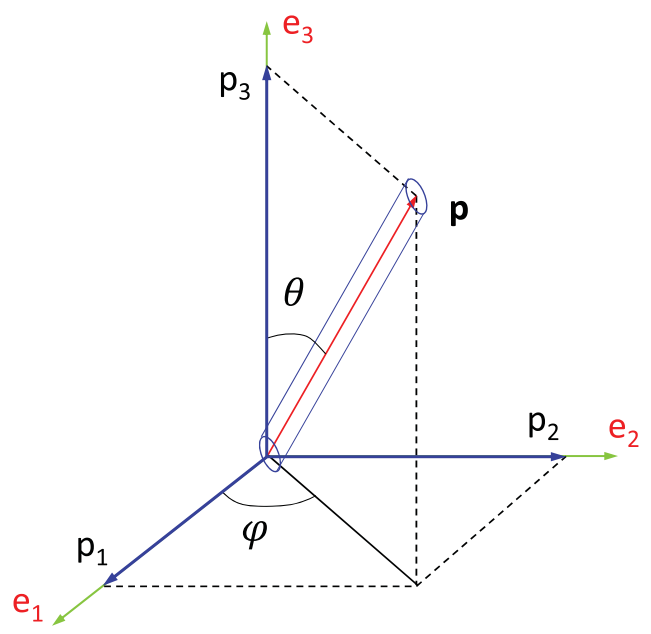

FIG. 6. (Color online) The fiber definition in the polar coordinates $(\varphi, \theta)$. scales of a few millimeters or more, a relative deviation from $\theta=90^{\circ}$ can occur, depending on the manufacturing process. $^{32}$

In Table III the calculated transport properties in the generated random fibrous materials for fibers increasingly inclined relative to the $x-y$ plane are displayed (increasingly aligned relative to the $z$ axis). Figure 7 shows transport parameters computed for a constant porosity $(\phi \sim 0.900$ $\pm 0.003)$ as a function of the statistically averaged fibers orientation $\left(0 \leq \Omega_{z z} \leq 1\right)$. The effect of angular orientation on transport parameters is documented in Fig. 7, in which the mean quantities are displayed for ten different generations of random fibrous samples, whose reconstructions satisfy a statistically averaged angular orientation, given in terms of $\Omega_{z z}$ (Table III). We varied the values of $\Omega_{z z}$ by adjusting the mean $\mu_{\theta}$ and standard deviation $\sigma_{\theta}$ of a distribution supposed to be normal for the angle $\theta$. The angle $\varphi$ is supposed to follow a uniform distribution between 0 and $2 \pi$. These results indicate that the chosen value of the inclination parameter $\left(0 \leq \Omega_{z z} \leq 1\right)$ modifies $k_{0}$ significantly: the permeability for the strongly aligned networks is higher than the permeability for the moderately aligned networks, up to a factor of 2.9 [Table III and Fig. 7(a)]. In addition, the dependence of permeability on the porosity at constant inclination was also verified. The fiber diameters were slightly modified in order to provide several values for the porosity and to keep the angular orientation of the fibers unchanged, for each of the ten reconstructed samples at $\Omega_{z z}=0.054$. Obviously, when the fiber diameters are slightly increased/decreased in the numerical model with the same cubic box dimension, this gives a lower/higher porosity, respectively. As the porosity increases with a relative difference of $0.42 \%$, the numerical model displays a permeability of $21.411 \pm 3.053 \times 10^{-11}$ $\mathrm{m}^{2}$; and a porosity decrease of $0.42 \%$ is associated with a permeability of $14.227 \pm 0.546 \times 10^{-11} \mathrm{~m}^{2}$. The data in Table III were also employed to calculate $\Lambda^{\prime} / \Lambda$, Fig. 7(b). For $\Omega_{z z}=1$, the fibers were perfectly aligned in the direction of wave propagation such that the porous structure is comparable to a straight cylinder with constant cross section for which $\Lambda^{\prime}=\Lambda\left(\Lambda^{\prime} / \Lambda=1\right)$. The $\Lambda^{\prime} / \Lambda$ values increase with the misalignment of fibers. At $\Omega_{z z}=0$, the fibers are perpendicular to the direction of wave propagation, and the theoretical result ${ }^{2} \Lambda^{\prime}=2 \Lambda$ derived for dilute fibers $(\phi \rightarrow 1)$ is numerically recovered, because the velocity field interactions created by pairs of adjacent fibers can be neglected. 

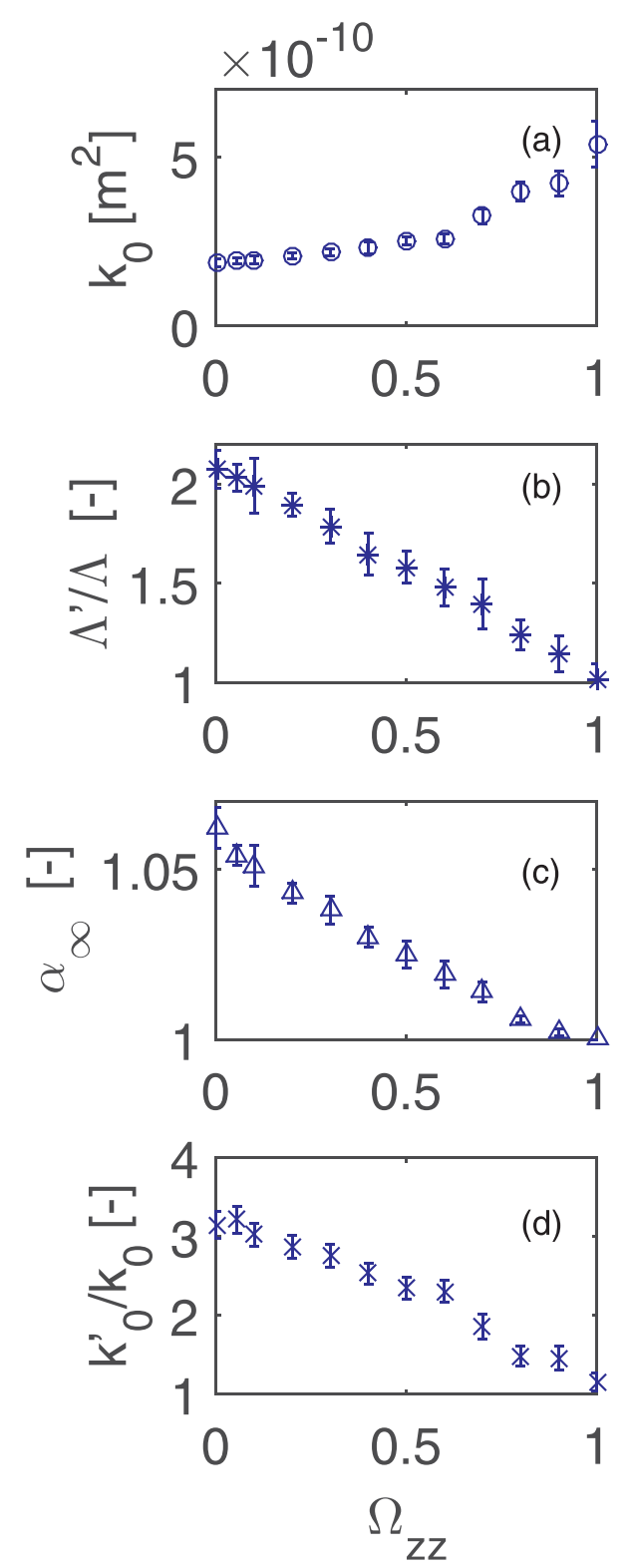

FIG. 7. (Color online) (a) The permeability $k_{0}$ as a function of the statistically averaged inclination of fibers as described by $\Omega_{z z}$ [see Eq. (20) for details], (b) ratio $\Lambda^{\prime} / \Lambda$ as a function of $\Omega_{z z}$, (c) tortuosity as a function of $\Omega_{z z}$, and (d) ratio $k_{0}^{\prime} / k_{0}$ as a function of $\Omega_{z z}$.

These results also show that the alignment of fibers affects tortuosity, Fig. 7(c). The $\alpha_{\infty}$-values decrease with the alignment of fibers. At a given $\phi$, the misaligned fiber networks have a larger $\alpha_{\infty}$ value than the aligned networks, and that difference increases with decreasing porosity. Additionally, to analyze the influence on tortuosity we carried out computations of transport parameters using a constant orientation of fibers close to the experimental configuration $\left(\Omega_{z z} \approx 0.054\right)$ and lower or higher porosity values (Fig. 8). With these porosity parameters, the calculated mean values of $\alpha_{\infty}$ increased proportionally with the diminution of the corresponding values in Fig. 7(c) $\left(\alpha_{\infty}=1.054 \pm 0.003\right.$ with $\phi=0.962$, and $\alpha_{\infty}$ $=1.091 \pm 0.013$ with $\phi=0.946$; for $\phi=0.987$, the $\alpha_{\infty}$-value was $=1.022 \pm 0.003$ ). These results indicate that tortuosity is a function of the number of fibers and of the fiber diameters at a given orientation of fibers. This change in tortuosity, however, turns out to be insignificant compared to the change in

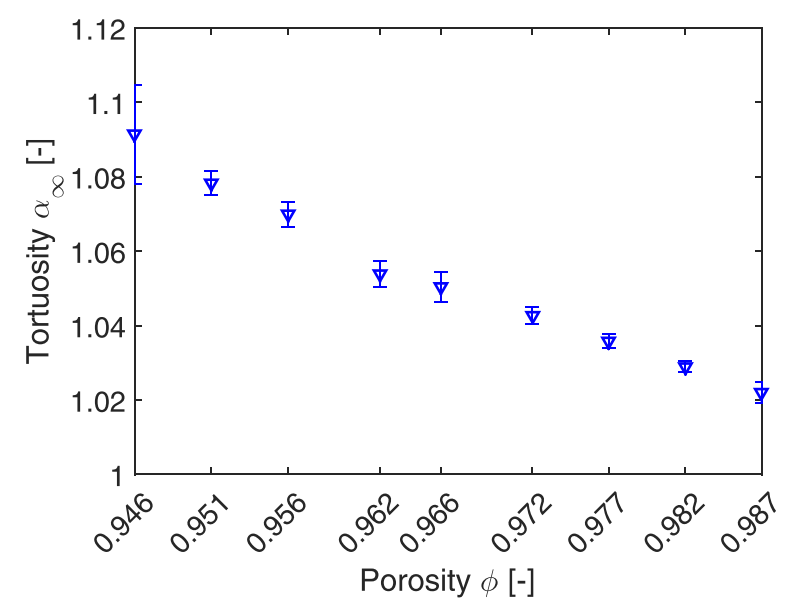

FIG. 8. (Color online) Tortuosity $\alpha_{\infty}$ as a function of porosity $\phi$ for a given orientation of fibers $\left(\Omega_{z z}=0.054\right)$ obtained by slightly modifying the fiber diameters.

other parameters. It also appears that combined simulations of viscous fluid flow and diffusion can be used to determine information about the orientation state of fibers. In fact, it was demonstrated that an inequality between $k_{0}$ and $k_{0}^{\prime}$ holds for all porous microgeometries, $k_{0}^{\prime} \geq k_{0} \cdot{ }^{33}$ When the fluid velocity is parallel to the direction of the fibers, the thermal and viscous permeabilities coincide: $k_{0}^{\prime} \simeq k_{0}$ (at $\Omega_{z z}=1$ ). Because $k_{0}^{\prime}$ is independent of the angular orientation state of fibers, and the viscous permeability $k_{0}$ decreases with an increasing misalignment of fibers, the ratio $k_{0}^{\prime} / k_{0}$ increases with the misalignment of fibers $\left(k_{0}^{\prime} / k_{0} \simeq 1\right.$ for $\Omega_{z z}=1$ and $k_{0}^{\prime} / k_{0}>3$ for $\Omega_{z z}=0$ ); Table III. In future works, it will be interesting to carry out analogous transport phenomena studies for disordered fibrous media to identify configurations which can yield enhanced acoustic properties. It would also be interesting to compare the results obtained during this work to complementary approaches using measurements and inverse estimates to identify the full permeability tensor of fibrous materials. ${ }^{34}$

\section{DISCUSSION}

This work reports results obtained for elementary transport phenomena in random fibrous media. A new class of sustainable acoustic materials, i.e., fibrous media made from milkweed Asclepias and bicomponent fibers, was modeled to determine their sound absorbing properties. The 3D geometry of the fibrous material was characterized based on data collected from SEM images, and the geometrical model was reconstructed using a fixed-generation protocol. The combination of a computer-generation protocol with fiber orientation distributions allowed us to study fibrous networks with emphasis on the influence of the fiber orientation distributions. In a transversely isotropic numerical model that allowed us to generate isoporous fiber webs, we explored the effect of fiber orientation, varying the velocity of the air from perpendicular to parallel to the direction of the fibers.

The calculation of transport properties was carried out using a FE scheme. It involves Stokes flow, potential flow, and diffusion-controlled reactions, which can be solved routinely in the reconstructed fibrous samples. Our results demonstrate that the values of the transport and acoustic 
coefficients in the investigated fiber webs are determined by their porosity $\phi$, the geometrical characteristics of the constitutive fibers, and by the degree of alignment of fibers as compared to the direction of wave propagation. At a given porosity $\phi$, the relative difference between $k_{0}$ values in transversely isotropic, moderated aligned, and aligned fibers webs may reach $285 \%$. The permeability coefficient computed by FEM was compared with $k_{0}$ values derived from direct standard measurements in the reconstructed fibrous sample, with a good agreement. The relative difference between the computed and measured values of the permeability was $1.66 \%$, without any fitting parameter (Table II). The use of the remaining transport coefficients allows for the estimation of sound absorbing properties directly from information on the geometrical structure of a fiber web.

\section{CONCLUSION}

Because the orientation of the fibers can be tuned from a state where air velocity is perpendicular to the direction of the fibers to the case where it is fluid velocity is parallel to the direction of the fibers, most of the transport properties can be modified.

For the studied fibrous sample, corresponding to simulated solid fiber webs with an open porosity of $\phi_{s}=0.9$, it was shown that the permeability significantly increases when the fibers are oriented parallel to the air velocity with a typical ratio of approximately three (when compared with the calculated $k_{0}$ values for the fibers lying in the planes perpendicular to the air velocity).

It was also shown that when the fibers' statistically averaged orientation angle (the ensemble average of $\theta$ defined in Fig. 6 relative to the air velocity) varies from $\pi / 2$ to zero, the length scales ratio $\Lambda^{\prime} / \Lambda$ decreases from two to one, in agreement with the known theoretical bounds [potential flow solution for a dilute fiber orthogonal to the flow direction, $\Lambda^{\prime}=2 \Lambda,{ }^{2}$ versus sound propagation in uniform tubes, $\Lambda^{\prime}=\Lambda$ (Ref. 20)].

Study of the viscous fluid flow and diffusion controlled reactions revealed quantitative agreement between the viscous permeability $k_{0}$ and the thermal permeability $k_{0}^{\prime}$, as long as the fibers are oriented parallel to the flow direction. Because the thermal permeability is insensitive to fiber orientation, the ratio $k_{0}^{\prime} / k_{0}$ combined with the rigorous upper bound $k_{0}^{\prime} \geq k_{0}$ can be used as an estimate of the statistically averaged deviation of the fibers' through-plane angle from zero.

The approximation $\alpha_{\infty} \approx 1$ appears to be valid over the entire range of angular orientations and porosities studied here. However, a relatively small increase in the tortuosity was observed when $\Omega_{z z}$ decreases.

The generalization and automatization of the approach to a large class of fibrous materials will be part of our further work. Extension to elastic properties will require the modeling of the connections between fibers. The proposed approach is extendable in principle to any physical property of fibrous media. Thus, the presented approach provides a straightforward routine to evaluate the effective properties of fibrous media and to analyze structure-property-manufacturing relationships in general.

\section{ACKNOWLEDGMENTS}

This work was partly supported by a grant of the Natural Sciences and Engineering Research Council of Canada (NSERC) whose support is gratefully acknowledged. Partial support for this work was also provided by Université Paris-Est (support for mobility grants from the ED SIE). We thank M. H. Alexandre, B. Stewart, and A. Hurrell for English editing assistance. The authors would like to thank two anonymous reviewers and the Associate Editor, K. V. Horoshenkov, for their judicious comments and suggestions which have certainly contributed in enhancing the quality and clarity of this paper.

\section{APPENDIX A: LOCATION OF THE SEM IMAGES}

The locations at which the SEM images were acquired are obtained using a procedure described here. Briefly, to acquire the pictures, seven cubic samples whose lateral dimensions were on the order of $4 \mathrm{~mm}$ were (destructively) collected in the bulk of the studied cylindrical sample $(44.5 \mathrm{~mm}$ in diameter) after acoustical testing. They were collected using an $8 \mathrm{~mm}$ square grid. Three of them were aligned along a horizontal line passing through the center of the cylinder, with a sample at the center, and equally spaced by a distance of $16 \mathrm{~mm}$ (Fig. 9). Two samples were selected according to the upper (respectively, lower) horizontal line, and placed by choosing a vertical line for which no sample was already extracted. Then, for one horizontal and one vertical plane of each cubic sample, four SEM images were acquired such as to span the overall surface.

\section{APPENDIX B: DERIVATION OF THE TOTAL FIBERS VOLUME}

In this appendix we briefly detail how the values of the total fibers' volumes $V_{a}$ and $V_{b}$ were derived from the knowledge of the targeted porosity $\phi$, and the ratio between bicomponent and Asclepias fibers $L_{b} / L_{a}$. The Asclepias and bicomponent fiber volumes are, respectively, equal to

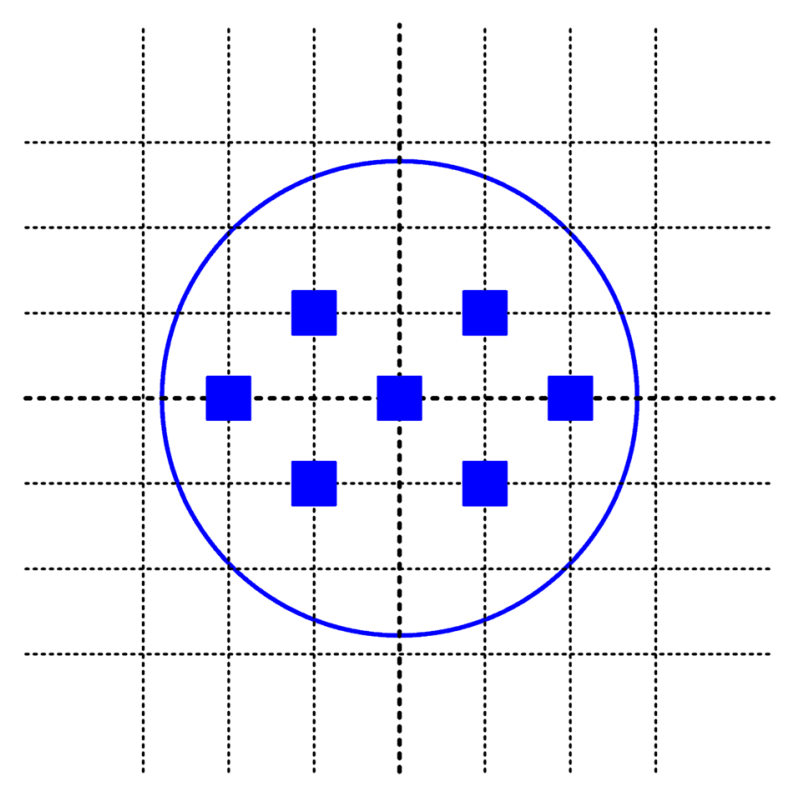

FIG. 9. (Color online) Location of the SEM images. 


$$
\begin{aligned}
V_{a} & =\pi\left[r_{a}^{2}-\left(r_{a}-t\right)^{2}\right] L_{a}, \\
V_{b} & =\pi r_{b}^{2} L_{b} .
\end{aligned}
$$

Therefore

$$
V_{b}=\frac{L_{b}}{L_{a}} \frac{r_{b}^{2} V_{a}}{r_{a}^{2}-\left(r_{a}-t\right)^{2}} .
$$

Consequently, the calculated porosity is

$$
\phi=1-\frac{V_{a}+V_{b}}{L^{(i) 3}} .
$$

Introducing Eq. (B3) into Eq. (B4) gives Eqs. (1) and (2).

\section{APPENDIX C: ESTIMATION OF THE ELASTIC PARAMETERS FROM AN AXISYMMETRICAL FE POROELASTIC MODEL}

In this appendix we explore how an axisymmetrical FE model with a Biot formulation can be used to identify the apparent elastic properties of the fibrous material under study. For further details on such poroelastic inversion based on an axisymmetrical FE model, see Ref. 28. Unlike the transport properties that can be directly determined from a basic set of elementary geometrical information, the determination of the elastic properties required adjustment such that the TL curves become comparable to those measured experimentally. To better replicate the mounting conditions $^{28}$ of the sample in the tube (due to friction, the cylindrical face of the sample was constrained by the tube wall), an appropriate axial motion constrain was imposed in the FE model. A close match between the sound transmission values in the experiment and in the poroelastic model confirms that the elasticity of the fibrous structure is an essential feature in capturing the physics of sound insulation, Fig. 5(b). The adjusted elastic properties of the corresponding poroelastic fibrous samples are $E=280000 \mathrm{~N} \mathrm{~m}^{-2}, \nu=0, \eta=0.01$, where $E, \nu$, and $\eta$, respectively, account for the Young's modulus, Poisson's ratio, and damping loss factor of the bounded samples inside an impedance tube of $44.5 \mathrm{~mm}$ in diameter. This triplet of parameters represents the apparent elastic behavior for a fibrous, yet bounded material. The Young's modulus appears to be relatively high when compared to standard fibrous materials. This has to be attributed to an effective rigidity due to boundary conditions, and also to the fact that the fibrous media was assumed to be isotropic whereas an assumption of transverse isotropy should better replicate the elastic behavior. The values corresponding to the remaining elastic parameters, $\nu$ and $\eta$, are classical for fibrous materials. For glass wool of density $30 \mathrm{~kg} / \mathrm{m}^{3}$, Tarnow $^{29}$ reported a constant complex Young's modulus $E_{3}{ }^{*}=12(1+0.05 j) \mathrm{kPa}$ in the direction perpendicular to which the fibers are laid (longitudinal direction). In the direction parallel to which the fibers are laid (transverse direction), the complex Young's modulus $E_{1}{ }^{*}$ was found to increase with frequency. At $20 \mathrm{~Hz} E_{1}{ }^{*}=1.5(1+0.01 j) \mathrm{MPa}$, and at $160 \mathrm{~Hz} E_{1}{ }^{*}=2.6(1+0.06 j) \mathrm{MPa}^{29}$ Obviously, the real part of the Young's modulus, corresponding to the studied sample mounted in an impedance tube, satisfies the inequality, $E_{3}<E<E_{1}$, where $E_{1}$ and $E_{3}$, respectively, account for the real parts of $E_{1}{ }^{*}$ and $E_{3}{ }^{*}$. This suggests that, when mounted in a tube of small diameter, the longitudinal and transverse elastic coefficients are strongly coupled, which has the effect of shifting the effective longitudinal Young's modulus toward the value of the transverse Young's modulus. Properly modeling the fiber-to-fiber interactions is intended to be the topic of a forthcoming paper.

${ }^{1} \mathrm{~K}$. Attenborough, "The influence of microstructure on propagation in porous fibrous absorbents," J. Sound Vib. 16, 419-442 (1971).

${ }^{2}$ J.-F. Allard and Y. Champoux, "New empirical equations for sound propagation in rigid frame fibrous materials," J. Acoust. Soc. Am. 91, 3346-3353 (1992).

${ }^{3}$ B. Castagnède, A. Aknine, B. Brouard, and V. Tarnow, "Effects of compression on the sound absorption of fibrous materials," Appl. Acoust. 61, 173-182 (2000).

${ }^{4}$ M. T. Pelegrinis, K. V. Horoshenkov, and A. Burnett, "An application of Kozeny-Carman flow resistivity model to predict the acoustical properties of polyester fiber," Appl. Acoust. 101, 1-4 (2016).

${ }^{5}$ M. M. Tomadakis and T. J. Robertson, "Viscous permeability of random fiber structures: Comparison of electrical and diffusional estimates with experimental and analytical results," J. Comp. Mat. 39, 163-188 (2005).

${ }^{6}$ J. F. Allard and N. Atalla, Propagation of Sound in Porous Media: Modelling Sound Absorbing Materials, 2nd ed. (Wiley, Chichester, United Kingdom, 2009), 358 pp.

${ }^{7}$ K. Schladitz, S. Peters, D. Reinel-Bitzer, A. Wiegmann, and J. Ohser, "Design of acoustic trim based on geometric modeling and flow simulation for non-woven," Comp. Mat. Sci. 38, 56-66 (2006).

${ }^{8}$ C. Jensen and R. Raspet, "Thermoacoustic properties of fibrous materials," J. Acoust. Soc. Am. 127, 3470-3484 (2010).

${ }^{9}$ C. Peyrega and D. Jeulin, "Estimation of acoustic properties and of the representative volume element of random fibrous media," J. Appl. Phys. 113, 104901 (2013).

${ }^{10} \mathrm{P}$. M. Adler and J.-F. Thovert, "Real porous media: Local geometry and macroscopic properties,” Appl. Mech. Rev. 51, 537-585 (1998).

${ }^{11}$ D. L. Johnson, J. Koplik, and R. Dashen, "Theory of dynamic permeability and tortuosity in fluid-saturated porous media," J. Fluid Mech. 176, 379-402 (1987).

${ }^{12}$ D. Lafarge, P. Lemarinier, J. F. Allard, and V. Tarnow, "Dynamic compressibility of air in porous structures at audible frequencies," J. Acoust. Soc. Am. 102, 1995-2006 (1997).

${ }^{13}$ Y. Salissou and R. Panneton, "Pressure/mass method to measure open porosity of porous solids," J. Appl. Phys. 101, 124913 (2007).

${ }^{14}$ C. Perrot, F. Chevillotte, M. T. Hoang, G. Bonnet, F. X. Bécot, L. Gautron, and A. Duval, "Microstructure, transport, and acoustic properties of open-cell foam samples: Experiments and three-dimensional numerical simulations," J. Appl. Phys. 111, 014911 (2012).

${ }^{15} \mathrm{M}$. Avellaneda and S. Torquato, "Rigorous link between fluid permeability, electrical conductivity, and relaxation times for transport in porous media," Phys. Fluids A 3, 2529-2540 (1991).

${ }^{16} \mathrm{~J}$. Rubinstein and S. Torquato, "Diffusion-controlled reactions: Mathematical formulation, variational principles, and rigorous bounds," J. Chem. Phys. 88, 6372-6380 (1988).

${ }^{17} \mathrm{COMSOL}$, Multiphysics user's guide, Comsol AB, v5.1.

${ }^{18}$ T. Kanit, S. Forest, I. Galliet, V. Mounoury, and D. Jeulin, "Determination of the size of the representative volume element for random composites: Statistical and numerical approach," Int. J. Solids Struct. 40, 3647-3679 (2003).

${ }^{19} \mathrm{C}$. Boutin and C. Geindreau, "Periodic homogenization and consistent estimates of transport parameters through sphere and polyhedron packings in the whole porosity range," Phys. Rev. E 82, 036313 (2010).

${ }^{20}$ Y. Champoux and J.-F. Allard, "Dynamic tortuosity and bulk modulus in air-saturated porous media," J. Appl. Phys. 70, 1975-1979 (1991).

${ }^{21}$ R. Panneton, "Comments on the limp frame equivalent fluid model for porous media,” J. Acoust. Soc. Am. 122, EL217-EL222 (2007).

${ }^{22}$ M. R. Stinson and G. A. Daigle, "Electronic system for the measurement of flow resistance," J. Acoust. Soc. Am. 83, 2422-2428 (1988). 
${ }^{23}$ N. Atalla, R. Panneton, and P. Debergue, “A mixed displacement-pressure formulation for poroelastic materials," J. Acoust. Soc. Am. 104, 1444-1452 (1998).

${ }^{24} \mathrm{X}$. Olny and C. Boutin, "Acoustic wave propagation in double porosity media," J. Acoust. Soc. Am. 114, 73-89 (2003).

${ }^{25} \mathrm{R}$. Panneton and X. Olny, "Acoustical determination of the parameters governing viscous dissipation in porous media," J. Acoust. Soc. Am. 119, 2027-2040 (2006).

${ }^{26} \mathrm{X}$. Olny and R. Panneton, "Acoustical determination of the parameters governing thermal dissipation in porous media," J. Acoust. Soc. Am. 123, 814-824 (2008).

${ }^{27}$ O. Doutres, Y. Salissou, N. Atalla, and R. Panneton, "Evaluation of the acoustic and non-acoustic properties of sound absorbing materials using a three-microphone impedance tube," Appl. Acoust. 71, 506-509 (2010).

${ }^{28}$ K. Verdiere, R. Panneton, N. Atalla, and S. Elkoun, "Inverse poroelastic characterization of open-cell porous materials using an impedance tube," SAE Technical Paper No. 2017-01-1878, in Proceedings of the SAE 2017 World Congress and Exhibition (Detroit, Michigan, 2017), p. 7.
${ }^{29} \mathrm{~V}$. Tarnow, "Dynamic measurements of the elastic constants of glass wool," J. Acoust. Soc. Am. 118, 3672-3678 (2005).

${ }^{30}$ S. G. Advani and C. L. Tucker, "The use of tensors to describe and predict fiber orientation in short fiber composites," J. Rheol. 31, 751-784 (1987).

${ }^{31}$ T. Stylianopoulos, A. Yeckel, J. J. Derby, X.-J. Luo, M. S. Shephard, E. A. Sander, and V. H. Barocas, "Permeability calculations in threedimensional isotropic and oriented fiber networks," Phys. Fluids 20, 123601 (2008).

${ }^{32}$ S. Bergonnier, F. Hild, and S. Roux, "Digital image correlation used for mechanical tests on crimped glass wool samples," J. Strain Anal. Eng. 40, 185-197 (2005).

${ }^{33}$ S. Torquato, "Relationship between permeability and diffusion-controlled trapping constant of porous media," Phys. Rev. Lett. 64, 2644-2646 (1990).

${ }^{34} \mathrm{C}$. Van der Kelen and P. Göransson, "Identification of the full anisotropic flow resistivity tensor for multiple glass wool and melamine foam samples," J. Acoust. Soc. Am. 134, 4659-4669 (2013). 